\title{
In search for effective and safe drugs against SARS-CoV-2: Part II] The role of selected salts and organometallics of copper, zinc, selenium, and iodine food supplements
}

\author{
M. S. A. Abdel-Mottaleb1 and Yousra Abdel-Mottaleb2 \\ 1Department of Chemistry, Faculty of Science, Ain Shams University (ASU), Abbassia 11566, \\ Cairo, Egypt \\ 2Department of Pharmacology, Toxicology and Biochemistry, Faculty of Pharmaceutical \\ Sciences and Pharmaceutical Industries, Future University in Egypt (FUE), New Cairo 11835, \\ Egypt
}

Corresponding author: m.s.abdelmottaleb@sci.asu.edu.eg

ihttps://orcid.org/0000-0002-4437-7040

2https://orcid.org/0000-0002-4327-4920

\begin{abstract}
:
The COVID-19 pandemic continues to burden global public health and economics. With limited treatment choices and high-risk side effects, the search for improved treatment is crucial and urgently needed. The fundamental role of angiotensin-converting enzyme 2 (ACE2) in the pathogenesis of COVID-19 has been previously reported. In this framework, three organometallics and three inorganic food supplements were selected, and their binding to specific peptide sequences of the coronavirus S-protein: ACE2 interface-drug binding adduct were computed. The chosen molecules located themselves to achieve geometries of minimum energy resulting in limiting viral recognition of the host cells or disturbing the host-virus interactions. Electrophilicity and nucleophilicity indices, based on the HOMO - LUMO frontier orbitals, were successfully used to explain the simulation results. Zinc and copper-supplements act as electron donors (nucleophiles) towards the ACE2 (electrophile), whereas the S protein remains inert. Molecular iodine acts as a strong electron sink in all of its unstable adducts with the ACE2, or the S protein, and in its adduct with both. Iodine is a stand-in as an electron shield to the ACE2. Currently, exploiting iodine has not been attempted, and it has been forgotten; while it has been used successfully in the treatment of the Spanish flu that started early 1918 and killed 30 million people. The results obtained strongly suggest the beneficial use of iodine. Selenodiglutathione exhibits strong electrophilic property and turns the $\mathrm{S}$ protein into an electron sink virus in the presence of
\end{abstract}


ACE2, which behaves like an electron donor. Contrary to zinc gluconate and iodine, the presence of selenomethionine, copper sulfate, selenodiglutathione, or sodium selenite strengthens the ACE2 - S protein interaction. The results reported indicate the association of common food supplements to offer protection and/or treatment against coronavirus S-protein COVID-19. These findings indicate also that these simple methods could help with the fight against COVID-19. In vivo and in vitro experimental validation is now required.

Keywords: Viral S protein, ACE2, Food supplements, Organometallics, Frontier orbitals, Reactivity indices, Binding energy

\section{1- Introduction}

Trace elements such as copper, zinc, selenium, and iodine are micronutrients in our diet that are necessary to sustain optimal biological functions [1-3] and optimal immune responses to infections including viral ones [4].

Copper plays a fundamental role in immunity by participating in the development and differentiation of immune cells and a direct effect on viral infections [5]. Zinc participates in the maintenance of immune function, and its deficiency has been linked with an increased susceptibility to viral infections [6]. Another trace element, selenium, is necessary for many physiological functions [7]. Low selenium status has been associated with poor immune function, while a higher selenium concentration or selenium supplementation has shown antiviral effects [7].

Iodine is a general antiseptic that can cripple viruses, bacteria, fungi, and their spores. Iodine proved effective against a multitude of viruses such as influenza, herpes, chicken, and smallpox [8-14]. Studies have suggested the use of iodine orally to prevent/treat viral infections. This suggestion is backed by the already established use of iodine in the treatment of thyroid disorders [15].

Earlier studies on some drugs and nutraceuticals that show potential benefits in the treatment/prevention of COVID-19 pandemic have been proposed [16]. In this context, computational simulation of the interactions between an $\mathrm{S}$ protein sequence ( assumed to represent the virus) with the drugs and with ACE2 is applied to identify the binding energies of the formed adducts and the mechanism of the interactions as well as to guide the further development of 
promising molecules that could be easily utilized to take the virus down. Furthermore, recently, a molecular docking study was reported which identified small molecules that bind to either the isolated Viral S-protein at its host receptor region or to the S protein-human ACE2 interface using the world's most powerful supercomputer, SUMMIT [17]. Reactivity indices of the interacting molecules were not identified or discussed.

Here, we explored the potential of commercially available food supplements known to act as immunity enhancers towards the new virus SARS-CoV-2. Through our work reported here, we are exploring in a simplified way the binding capability of five drugs of some food supplement formulations to accelerate the discovery of improved treatment options, thus paving the way to a hopefully near future purge of the COVID-19 pandemic. The results presented are a first step towards the identification of the importance of the electronic characteristics such as electrophilicity $(\omega)$ (based on the chemical potential $(\mu)$ and the hardness $(\eta)$ indecis ) and nucleophilicity $(\mathrm{N})[18$ $24]$ of the drugs and utilization in treatments with potentially low-risk side effects relative to the current insufficient high risk treatment options against COVID-19.

\section{1- Simulation Methodology}

The equilibrium geometry of the interacting molecules was obtained by applying the molecular mechanics' method built-in Spartan 18 parallel suite (core > 16) package offered by www.Wavefun.com at reduced prices due to COVID-19 lockdown. Single-point computations are then applied to the molecular mechanically optimized equilibrium geometry with DFT using the well-known RB3LYP method with 6-31G(d) and LANL2DZ > Kr basis sets [25]. The method used is efficient and cost-effective for capturing information across biological scales. Other more expensive functionals such as wB97X-D [Long-range corrected hybrid density functional, the wB97X-D functional [26] includes empirical damped atom-atom dispersion corrections.] or higher basis sets did not result in any significant change in the results obtained.

The DFT-B3LYP method has been demonstrated to predict excellent geometries and energies [27]. The useful reaction field model used for solvation (water) is the conductor-like polarizable continuum model (CPCM), which performs a PCM (polarized continuum model) calculation [28]. 


\section{2- Results and Discussion}

In the present study, we followed the same procedure reported previously [16] to compute the binding energy (BE) using the well-known equation:

BEadduct $=$ Eadduct $-(\Sigma$ Einteracting species $)$

The results are summarized in Tables 1, 2, 3, and 4. Table 1 shows the frontier orbital HOMO -LUMO energy values and the well-known reactivity indices that reflect the electronic donoracceptor reactivity of the individual ligands and ACE2 [29]- S protein adduct [30, 31].

The stabilities of the adducts given in Tables 2, 3, and 4 stem from hydrogen bond formation and other electrostatic attractive/repulsive forces due to the HOMO - LUMO electron transfer capabilities between the interacting molecules. Figure 1 depicts the shapes of the ligands. Figures 2-5 represent the effect of the ligands on the donor - acceptor properties of the adducts with ACE2 and S protein.

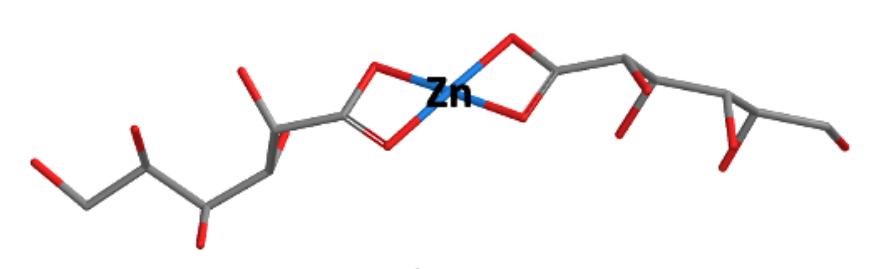

Zinc gluconate

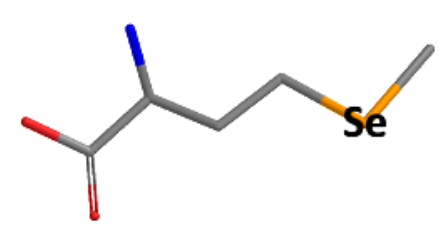

Selenomethionine
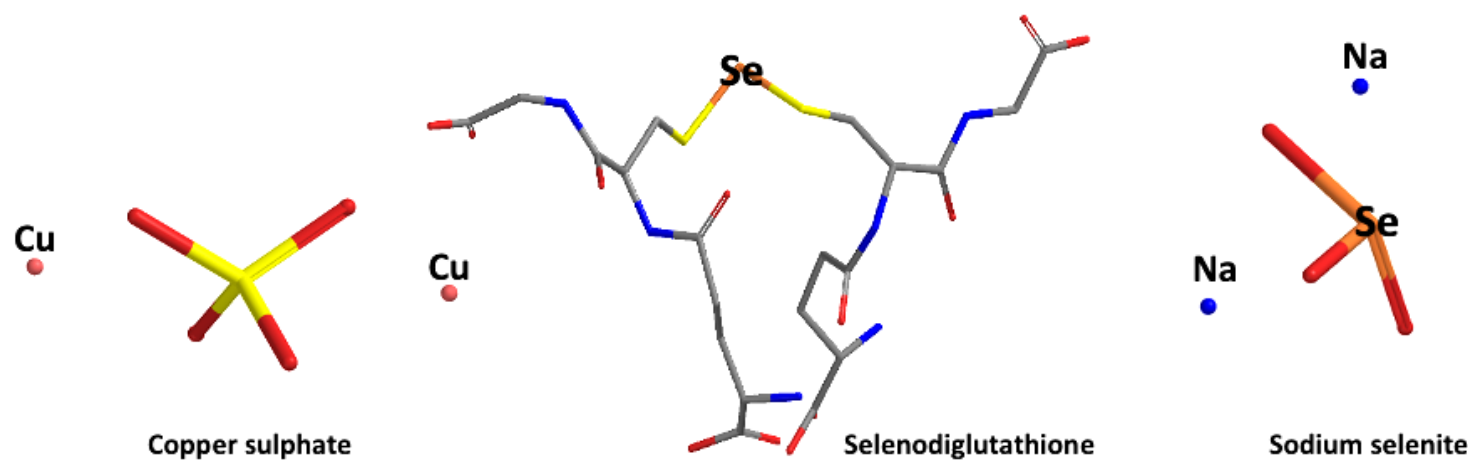

Figure 1. The chemical structures of the studied molecules (hydrogen atoms are removed for clarity). Iodine is a covalent diatomic molecule (not shown). 
Table 1-Reactivity indices of individual ligand molecules, ACE2, S protein, and ACE2 - S protein adduct

\begin{tabular}{c|c|c|c|c|c|c|c}
\hline FREE LIGANDS/Interacting proteins & $\mathrm{HO}$ & $\mathrm{LU}$ & $\mu$ & $\eta$ & $\omega$ & $\mathrm{N} / \mathrm{eV}$ & ID: ChemSpider \\
\hline Zinc gluconate & -5.54 & -1.02 & -3.28 & 2.26 & 2.38 & 5.65 & 391659 \\
\hline Selenomethionine & -5.76 & 0.03 & -2.86 & 2.89 & 1.42 & 5.43 & 14375 \\
\hline Copper sulfate & -3.80 & -1.20 & -2.50 & 1.30 & 2.40 & 7.39 & 118749 \\
\hline selenodiglutathione & -6.76 & -6.48 & -6.62 & 0.14 & 156.50 & 4.43 & 97171 \\
\hline Sodium selenite & -6.18 & -0.75 & -3.47 & 2.72 & 2.21 & 5.01 & 1059 \\
\hline iodine & -9.19 & -2.24 & -5.72 & 3.48 & 4.70 & 2.00 & 785 \\
\hline ACE2 & -5.97 & -0.93 & -3.45 & 2.52 & 2.36 & 5.22 & - \\
\hline S protein & -5.27 & -0.29 & -2.78 & 2.49 & 1.55 & 5.92 & - \\
\hline ACE2 - S protein & -5.35 & -0.91 & -3.13 & 2.22 & 2.21 & 5.84 & -
\end{tabular}

The electrophilicity index $\left(\omega=\mu_{2} /(2 * \eta)\right.$ of a ligand measures its ability to act as an electron sink. The higher its value, the stronger is the electron sink property of a ligand. The listed data reveal that the electrophilicity strength decreases in the order:

selenodiglutathione $>$ iodine $>$ copper sulfate $>$ zinc gluconate $>$ ACE2 $>$ sodium selenite $>$ S protein $>$ selenomethionine

The nucleophilicity index $\{21,22]$ decreases in the order: copper sulfate $>\mathrm{S}$ protein $>$ zinc gluconate $>$ selenomethionine $>$ ACE2 $>$ sodium selenite $>$ selenodiglutathione $>$ iodine

Both indices are generally consistent with each other and point to iodine and selenodiglutathione as the best electrophiles (acting as strong electron sinks). Figures 3 and 5 clearly indicate the role played by these materials as strong electron sinks. Interestingly, the nucleophilicity index of copper sulfate is manifested in its electron donor capability to ACE2 in the presence of S protein.

Table 2- HOMO - LUMO energies (eV) and binding energies, BE of the adducts: ACE2 - ligand

\begin{tabular}{c|c|c|c}
\hline ACE2 - LIGAND & HOMO & LUMO & BE/kcal/mol \\
\hline Zinc gluconate & -4.84 & -1.15 & +14.66 \\
\hline Selenomethionine & -5.91 & -0.95 & -13.13 \\
\hline Copper sulfate & -2.77 & -1.10 & -57.58 \\
\hline selenodiglutathione & -6.17 & -5.92 & -3.30 \\
\hline Sodium selenite & -5.82 & -0.88 & -23.18 \\
\hline iodine & -5.91 & -3.60 & +6.09 \\
\hline
\end{tabular}

It is clearly shown that the zinc gluconate and iodine molecules did not form stable complexes with ACE2 (see Table 2). Interestingly, ACE2 interacts with zinc gluconate as an electron acceptor, whereas it acts as an electron donor with iodine. This is due to the smaller 
energy gap between the HOMO of zinc gluconate and the LUMO of ACE2. The HOMO - LUMO energy gap in the case of the iodine - ACE2 adduct favors the above-mentioned donor-acceptor properties.

ACE2 shows the same electron acceptor property in the case of all other adducts with the ligands listed in Table 1. Inspection of Table 2 reveals that the order of stability of the ligand - ACE2 adducts decreases as follows: copper sulfate > sodium selenite > selenomethionine > selenodiglutathione > iodine> zinc gluconate.

Table 3-HOMO - LUMO energies (eV) and binding energies, BE of the adducts: S proteinligands

\begin{tabular}{c|c|c|c}
\hline S protein - LIGAND & HOMO & LUMO & BE/kcal/mol \\
\hline Zinc gluconate & -5.25 & -0.94 & -0.65 \\
\hline Selenomethionine & -5.25 & -0.29 & -1.09 \\
\hline Copper sulfate & -3.8 & -1.01 & -28.73 \\
\hline selenodiglutathione & -6.23 & -5.97 & -33.70 \\
\hline Sodium selenite & -5.3 & -0.68 & -16.95 \\
\hline iodine & -5.32 & -3.59 & +2.31 \\
\hline
\end{tabular}

Referring to Table 3, the BE of the ligand - S protein adduct stability decreases as follows: selenodiglutathione $>$ copper sulfate $>$ sodium selenite $>$ selenomethionine $>$ zinc gluconate $>$ iodine. $\mathrm{S}$ protein acts as a nucleophile for all ligands except in the case of copper sulfate, which enforces the $\mathrm{S}$ protein to exhibit an electron acceptor property. The results can be explained by the same argument of the HOMO - LUMO energy gap between the ligand and the S protein.

Table 4- HOMO - LUMO energies (eV) and binding energies, BE of the adducts: S protein ACE2 - ligands

\begin{tabular}{c|c|c|c}
\hline $\mathrm{S}$ protein -ACE2 - ligands & HOMO & LUMO & BE/kcal/mol \\
\hline Zinc gluconate & -5.1 & -1.10 & +36.97 \\
\hline Selenomethionine & -5.23 & -0.88 & -0.46 \\
\hline $\mathrm{Cu}_{2} \mathrm{SO}_{4}$ & -2.66 & -1.10 & -42.54 \\
\hline selenodiglutathione & -6.02 & -5.76 & -33.59 \\
\hline $\mathrm{Na}_{2} \mathrm{SeO}_{3}$ & -5.18 & -0.93 & -32.07 \\
\hline iodine & -5.35 & -3.60 & +7.53 \\
\hline
\end{tabular}


Noteworthy mentioning that the value of the BE of the complex ligand - ACE2 - S protein (Table 4) decreases in the order: copper sulfate > selenodiglutathione > sodium selenite > selenomethionine > iodine > zinc gluconate. This trend points to the importance of iodine and zinc gluconate as the most effective materials for destabilizing the adduct ACE2 - S protein. Moreover, the organometallic zinc gluconate deactivates the electron donor-acceptor of the $\mathrm{S}$ protein, as shown in Figure 2. Thus, it is obvious that the $\mathrm{S}$ protein becomes an inert species towards ACE2 in the presence of the zinc gluconate molecule. On the other hand, the iodine molecule exhausts the electron donor property of the $\mathrm{S}$ protein by acting as a strong electron sink (with the smallest $\mathrm{N}$ value and large $\omega$ value) that results in deactivates the electron donor or acceptor properties of ACE2 in the presence of S protein (see Figure 3).

Although copper sulfate strengthens the stability of the adduct, it turns the $\mathrm{S}$ protein into an inert species (see Figure 4). Consequently, whether the formed adduct is energetically stable or unstable, the HOMO - LUMO energy gap of the interacting species plays a key role in deactivating the electronic properties of one of the reactants.

Figure 5 shows that in the stable adducts of selenoglutathione with the ACE2 and S proteins, both selenoglutathione together with $\mathrm{S}$ protein are acting as electron sinks, while ACE2 acts as an electron donor, indicating that the ACE2 is still suffering by interactions with the S protein. Other ligands, in particular, the sodium selenite and selenomethionine are not involved in the HOMO LUMO interactions and did not prevent the ACE2 - S protein direct interactions. 


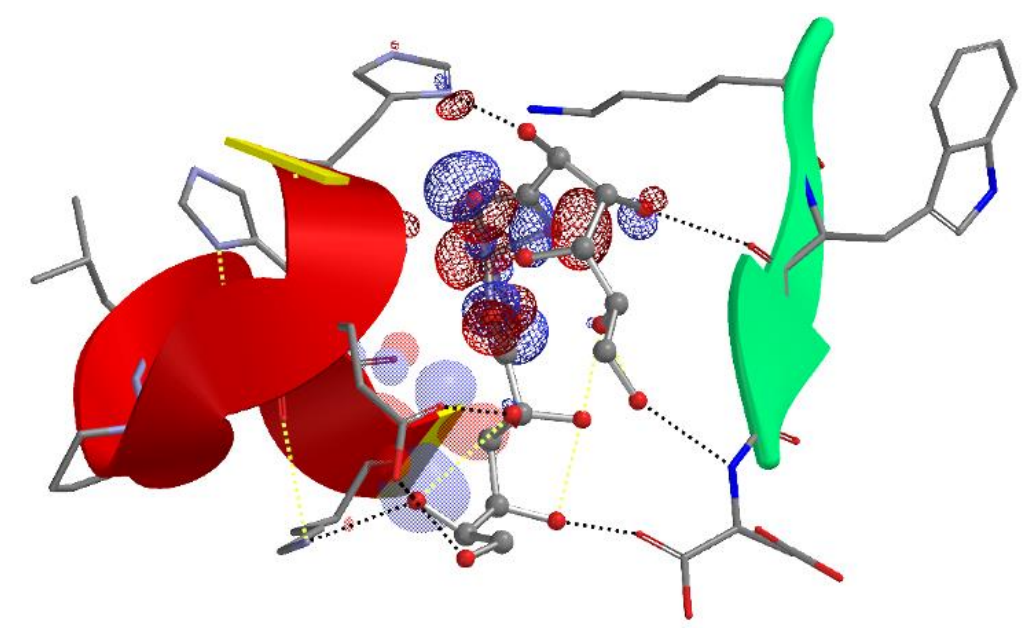

Figure 2. Zinc gluconate donates electrons via its HOMO (mesh) to the ACE2 LUMO (transparent) which acting as an electron sink in the presence of inert S protein. Unstable complex is formed with $\mathrm{BE}=36.97 \mathrm{k} \mathrm{Cal} / \mathrm{mol}$

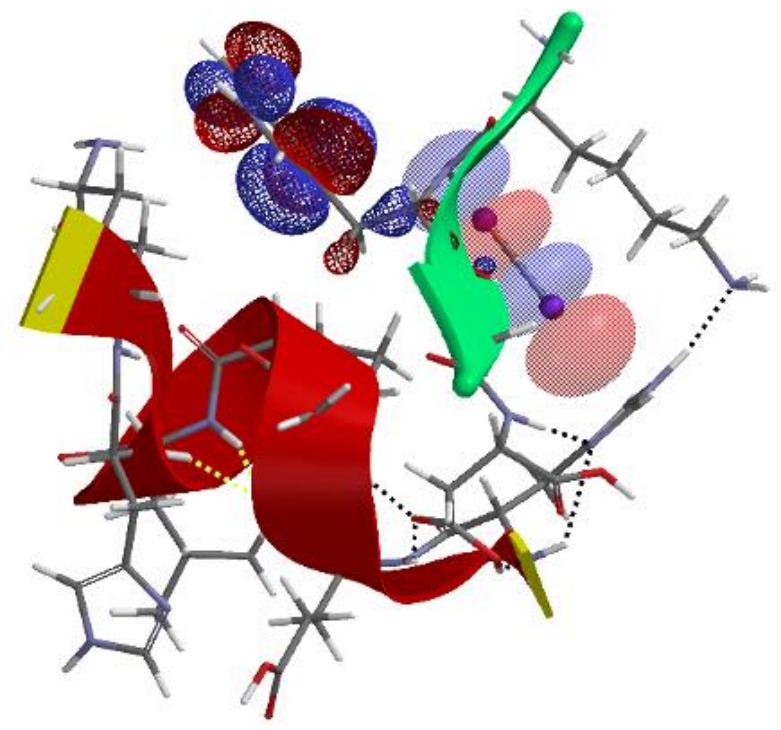

Figure 3. Iodine exhausts the electron donor property of the S protein by acting as a strong electron sink that deactivate the electron donor or acceptor properties of the ACE2 in the presence of S protein. 


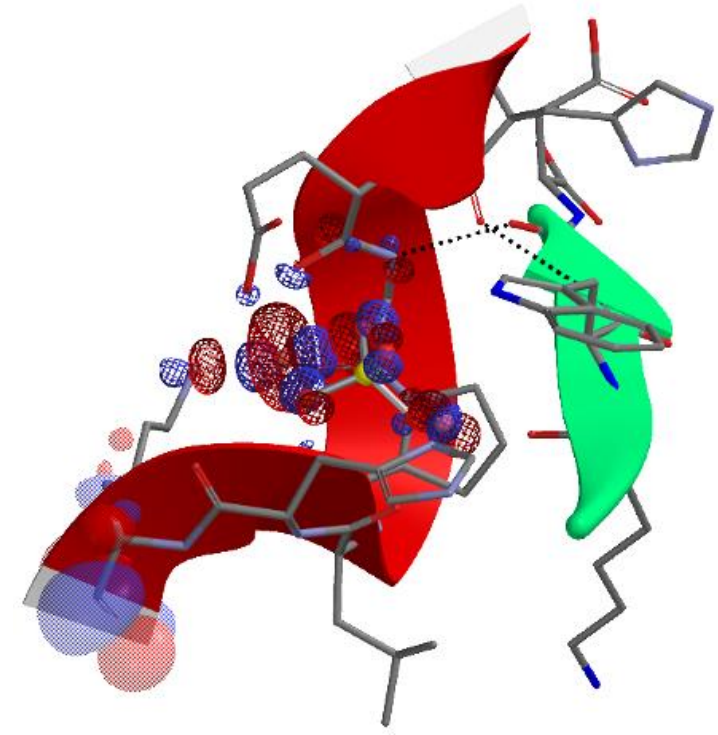

Figure 4. Copper sulphate (the nucleophile) turns $S$ protein inert in the presence of ACE2 (the electrophile). However, strong complex is formed with BE $=-42.54 \mathrm{k} \mathrm{Cal} / \mathrm{mol}$

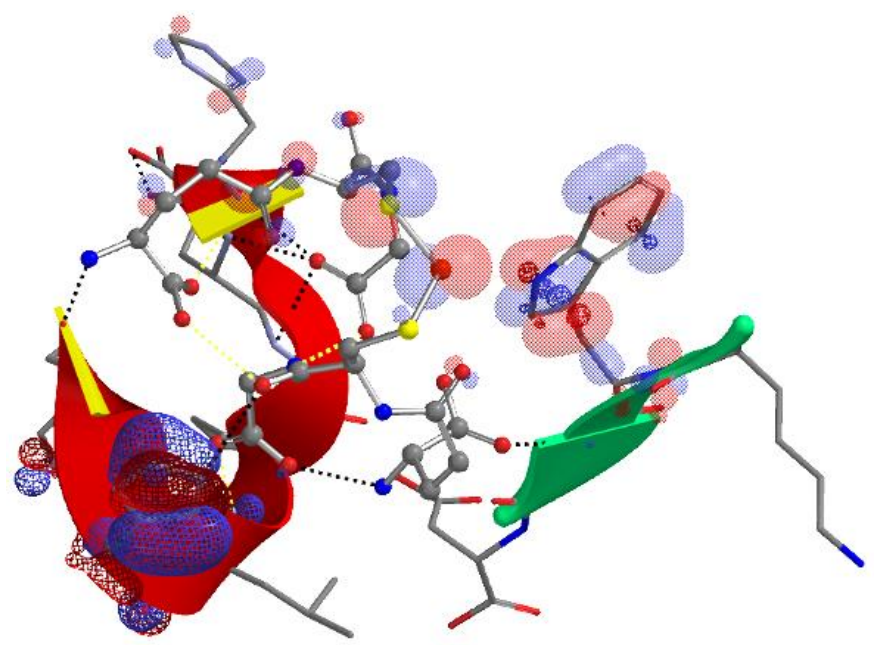

Figure 5. Selenoglutathione together with $S$ protein are acting as electron sinks (the electrophiles, transparent LUMO) while ACE2 acting as an electron donor (mesh)

Iodine has been exploited as a prophylactic and therapeutic agent in the treatment of diseases caused by bacteria, viruses, and fungi, and to sanitize eating utensils. Iodine kills within seconds of bacteria, viruses [32, 33] fungi, protozoa, and even spores of 
bacteria and fungi, including anthrax spores. Iodine has been used successfully and efficiently against influenza, herpes, smallpox, and chicken pox viruses [33].

\section{3- Conclusions}

We simulated the interactions of model simple peptides that were chosen to represent the S protein and the ACE2 enzyme with five inorganic and organometallic ligands that are found in some food supplements. Namely, the materials are copper sulfate, selenodiglutathione, sodium selenite, selenomethionine, iodine, and zinc gluconate. The binding energies of these materials with both ACE2 and S protein were calculated. Exothermic and endothermic reactions were predicted. The adducts formed with the iodine and zinc gluconate molecules are endothermic, revealing unstable complex formation. Interestingly, the $S$ protein is neutralized (becomes inactive towards ACE2) because of the presence of the strong electron donor ligands of the zinc gluconate and the copper sulfate. Both ligands are considered as electron shielding agents for ACE2. On the other hand, the iodine molecule exhausts the electron donor property of the S protein by acting as a strong electron sink that results in deactivating the electron donor or acceptor properties of the ACE2 in the presence of $S$ protein. The electrophilicity and nucleophilicity indices were successfully used to explain the reactivities of the interacting molecules. The results obtained led us to conclude that using iodine, zinc gluconate, and/or copper sulfate effectively neutralizes the influence of the S protein on ACE2. In vitro and in vivo verification is highly required.

\section{Conflict of interests}

There are no conflicts of interest to declare. All data are available to share upon request from the corresponding author. 


\section{References}

[1] R.L. Bailey, K.P. West, Jr., R.E. Black, The epidemiology of global micronutrient deficiencies, Ann Nutr Metab 66 Suppl 2 (2015) 22-33.

[2] D.C. McMillan, D. Maguire, D. Talwar, Relationship between nutritional status and the systemic inflammatory response: micronutrients, Proc Nutr Soc 78(1) (2019) 56-67.

[3] R. Jayawardena, P. Sooriyaarachchi, M. Chourdakis, C. Jeewandara, P. Ranasinghe, Enhancing immunity in viral infections, with special emphasis on COVID-19: A review, Diabetes Metab Syndr 14(4) (2020) 367-382.

[4] M.N. Rashed, The role of trace elements on hepatitis virus infections: a review, J Trace Elem Med Biol 25(3) (2011) 181-7.

[5] C. Li, Y. Li, C. Ding, The Role of Copper Homeostasis at the Host-Pathogen Axis: From Bacteria to Fungi, Int J Mol Sci 20(1) (2019).

[6] S.A. Read, S. Obeid, C. Ahlenstiel, G. Ahlenstiel, The Role of Zinc in Antiviral Immunity, Adv Nutr 10(4) (2019) 696-710.

[7] M.P. Rayman, Selenium and human health, Lancet 379(9822) (2012) 1256-68.

[8] L. Gershenfeld, Iodine as a virucidal agent, J Am Pharm Assoc Am Pharm Assoc 44(3) (1955) 177-82.

[9] L. Gershenfeld, Povidone-iodine (PVP-I) as a trichomonacide, Am J Pharm Sci Support Public Health 134 (1962) 324-31.

[10] L. Gershenfeld, Povidone-iodine (PVP-I) as a vaginal microbicide, Am J Pharm Sci Support Public Health 134 (1962) 278-91.

[11] L. Gershenfeld, Povidone-iodine as a sporicide, Am J Pharm Sci Support Public Health 134 (1962) 78-81.

[12] L. Gershenfeld, W.B. Flagg, B. Witlin, Iodine as a tuberculocidal agent, Mil Surg 114(3) (1954) 172-83.

[13] L. Gershenfeld, B. Witlin, Iodine as an antiseptic, Ann N Y Acad Sci 53(1) (1950) 172-82.

[14] M. Eggers, Infectious Disease Management and Control with Povidone Iodine, Infect Dis Ther 8(4) (2019) 581-593.

[15] D. Derry, Iodine: the Forgotten Weapon Against Influenza Viruses, Thyroid Science 4(9) (2009) 1-5. 
[16] M. Sabry Abdel-Mottaleb and Yousra Abdel-Mottaleb, In Search for Effective and Safe Drugs Against SARS-CoV-2: Part I] Simulated Interactions Between Selected Nutraceuticals, ACE2 Enzyme and S Protein Simple Peptide Sequences., ChemRxiv (2020).

[17] M.S. Smith, Jeremy C. , Repurposing Therapeutics for COVID-19: Supercomputer-Based Docking to the SARS-CoV-2 Viral Spike Protein and Viral Spike Protein-Human ACE2 Interface, ChemRxiv (2020).

[18] P. Geerlings, F. De Proft, W. Langenaeker, Conceptual density functional theory, Chem Rev 103(5) (2003) 1793-873.

[19] I. Fleming, Frontier Orbitals and Organic Chemical Reactions, John Wiley and Sons, New York, 1976.

[20] L.R. Domingo, E. Chamorro, P. Perez, Understanding the reactivity of captodative ethylenes in polar cycloaddition reactions. A theoretical study, J Org Chem 73(12) (2008) 4615-24.

[21] L.R. Domingo, M. Rios-Gutierrez, P. Perez, Applications of the Conceptual Density Functional Theory Indices to Organic Chemistry Reactivity, Molecules 21(6) (2016).

[22] L.R. Domingo, Molecular Electron Density Theory: A Modern View of Reactivity in Organic Chemistry, Molecules 21(10) (2016).

[23] L.R. Domingo, A new C-C bond formation model based on the quantum chemical topology of electron density, RSC Adv. 4 (2014) 32415-32428.

[24] V.T.a.L.J. F. Zielinski, Condensed descriptors for reactivity: a methodological study, Chemical Physics Letters 527 (2012) 67-72.

[25] P.J.H.a.W.R. Wadt, Ab initio effective core potentials for molecular calculations - potentials for the transition-metal atoms Sc to Hg, J. Chem. Phys. 82 (1985) 270-83.

[26] J.D.a.H.-G. Chai, M., Long-range corrected hybrid density functionals with damped atomatom dispersion corrections, Physical Chemistry Chemical Physics 10(44) (2008) 6615-6620.

[27] B.A. D., Density-functional thermochemistry. III. The role of exact exchange, J. Chem. Phys 98 (1993) 5648-52.

[28] N.R. M. Cossi , G. Scalmani, V. Barone, , Energies, structures, and electronic properties of molecules in solution with the C-PCM solvation model, J. Comput. Chem. 24(6) (2003) 669-681. [29] W. Li, M.J. Moore, N. Vasilieva, J. Sui, S.K. Wong, M.A. Berne, M. Somasundaran, J.L. Sullivan, K. Luzuriaga, T.C. Greenough, H. Choe, M. Farzan, Angiotensin-converting enzyme 2 is a functional receptor for the SARS coronavirus, Nature 426(6965) (2003) 450-4. 
[30] Courtney Mycroft-West, Dunhao Su, Stefano Elli, Scott Guimond, Gavin Miller, Jeremy Turnbull, Edwin Yates, Marco Guerrini, David Fernig, Marcelo Lima, M. Skidmore, The 2019 coronavirus (SARS-CoV-2) surface protein (Spike) S1 Receptor Binding Domain undergoes conformational change upon heparin binding, BioRxiv (2020).

[31] Z. Li, A.C. Tomlinson, A.H. Wong, D. Zhou, M. Desforges, P.J. Talbot, S. Benlekbir, J.L. Rubinstein, J.M. Rini, The human coronavirus HCoV-229E S-protein structure and receptor binding, Elife 8 (2019).

[32] B. Carroll, The relative germicidal activity of triiodide and diatomic iodine. , J. Bacteriol. (69) (1955) 413-417

[33] L. Gershenfeld, Iodine. In Disinfection, Sterilization, and Preservation. , Les \& Febiger, Philadelphia, 1977. 